\title{
Accuracy limits on the description of the lowest $S$ excitation in the Li atom using explicitly correlated Gaussian basis functions
}

\author{
Monika Stanke, ${ }^{1,2}$ Jacek Komasa, ${ }^{3}$ Dariusz Kędziera, ${ }^{4}$ Sergiy Bubin,,${ }^{1}$ and Ludwik Adamowicz ${ }^{1,5}$ \\ ${ }^{1}$ Department of Chemistry, University of Arizona, Tucson, Arizona 85721, USA \\ ${ }^{2}$ Institute of Physics, Nicholas Copernicus University, ul. Grudziadzka 5, PL 87-100 Toruń, Poland \\ ${ }^{3}$ Quantum Chemistry Group, Faculty of Chemistry, A. Mickiewicz University, Grunwaldzka 6, 60-780 Poznań, Poland \\ ${ }^{4}$ Department of Chemistry, Nicholas Copernicus University, ul. Gagarina 7, PL 87-100 Toruń, Poland \\ ${ }^{5}$ Department of Physics, University of Arizona, Tucson, Arizona 85721, USA
}

(Received 31 August 2008; published 14 November 2008)

\begin{abstract}
We have performed very accurate quantum-mechanical calculations for the two lowest $S$ states of the lithium atom in order to determine the transition energy. In the nonrelativistic part of the calculations performed using the variational method, we explicitly included the nuclear motion. The nonrelativistic wave function was expanded in terms of explicitly correlated Gaussian functions. Next, this wave function was used to calculate the leading $\alpha^{2}$ relativistic correction ( $\alpha$ is the fine-structure constant) and the $\alpha^{3}$ QED correction. We also estimated the $\alpha^{4}$ QED correction by calculating its dominating component. The results obtained with Gaussians are compared with the most accurate results obtained recently with the Hylleraas-type basis functions.
\end{abstract}

DOI: 10.1103/PhysRevA.78.052507

PACS number(s): 31.15.ac, 31.15.aj, 31.30.jf

\section{INTRODUCTION}

In this work we continue our very accurate quantummechanical calculations of light atoms with an approach that utilizes explicitly correlated Gaussian (ECG) basis functions in expanding the nonrelativistic wave function. In our recent works [1-5] we have shown that with these types of functions we can calculate excitation energies for atoms with three and four electrons that agree very well with accurate spectroscopic data. To reach such accuracy the approach had to include treating the motion of the electrons and the motion of the nucleus on equal footing [the finite-nuclear-mass (FNM) approach [6-11]] and had to account for the leading relativistic and QED corrections. Since the explicitly correlated Gaussians depend on the distances between the electrons and the distances between the electrons and the nucleus, they can very effectively describe the correlation effects in the system. However, in general, Gaussians are less effective than the Hylleraas-type or Slater-type functions in this task because their cusp and long-range behaviors are not as good as of those other types of functions. The use of Gaussians, however, leads to much simpler integrals and, unlike the Hylleraas functions, these functions can be applied to systems with more than three electrons without an increase in the complexity of the algorithms for calculating the Hamiltonian matrix elements. In fact, the algorithms involving Gaussians can be derived and coded in a general form allowing calculations of atomic systems with an arbitrary number of electrons (the largest atomic systems we have considered so far were the Be atom [3] and the $\mathrm{Li}$ anion [4]). The only factor that limits the size of the calculated system is the computer power available for the calculations. This is not the case for the Hylleraas functions where the increase of the number of electrons from three to four leads to such an increase of the complexity of the calculations that a fourelectron atomic problem has not yet been attempted using those functions. An additional advantage of using Gaussians is that the Hamiltonian matrix elements calculated with those functions can be easily analytically differentiated with respect to the Gaussian exponential parameters and the energy gradient can be determined. Since the optimization of these parameters is key in obtaining high-accuracy results and the use of the gradient can significantly accelerate this optimization, this feature makes Gaussians very good basis functions for atomic calculations.

Very precise quantum-mechanical atomic calculations of bound ground and excited states of atoms can assist the interpretation of high-resolution experiments and contribute to refining fundamental physical constants. The modern experiments concerning atomic electronic transitions are now reaching the relative accuracy of $10^{-9}$. In order for the theoretical calculations to reproduce these results they need to account for even the smallest effects that originate from the electrostatic, relativistic, and QED interactions. For atoms with more than one electron, the most successful approach to perform very accurate calculations has been the method based on an effective Hamiltonian derived in the framework of the nonrelativistic QED method (NRQED) [12-14]. The zeroth-order level in this approach is the nonrelativistic Schrödinger equation, and the perturbation theory scheme is used to determine corrections describing the relativistic and QED effects. Those corrections are represented by quantities dependent on the fine-structure constant $\alpha(\alpha=1 / c)$ :

$$
E(\alpha)=E_{\mathrm{NR}}+\alpha^{2} E_{\mathrm{rel}}+\alpha^{3} E_{\mathrm{QED}}+\alpha^{4} E_{\mathrm{HQED}}+\cdots .
$$

The accuracy of the NRQED approach strongly depends on the accuracy of the solution of the nonrelativistic Schrödinger equation.

The leading terms in the expansion (1)-i.e. the nonrelativistic energy $E_{\mathrm{NR}}$, the relativistic correction $\alpha^{2} E_{\text {rel }}$, and the leading-order radiative correction $\alpha^{3} E_{\mathrm{QED}}$ - are well known since early works of Bethe and Salpeter [15], Araki [16], and Sucher [17]. An effective scheme to account for the $\alpha^{3}$ QED effects in light atoms was developed by Pachucki $[18,19]$. He also recently presented formulas for calculating the radiative 
correction of the order of $\alpha^{4}\left(\alpha^{4} E_{\mathrm{HQED}}\right)$ [19].

The purpose of this work is to study the performance of the Gaussians in comparison with the Hylleraas-type functions in the calculations of the two lowest $S$ states of the lithium atom. This is a good model case to study this question because very accurate calculations were done for this system with the Hylleraas basis functions [20-27]. The theoretical framework for the calculations performed in this work is provided by the expansion (1). The relativistic corrections are calculated using the nonrelativistic wave functions obtained in the FNM variational calculations with explicitly correlated Gaussian basis functions [1-3]. The QED correction of the order of $\alpha^{3}$ is calculated using the procedure developed by Pachucki and Komasa [28-31] also for the wave functions expanded in terms of explicitly correlated Gaussians, but obtained in infinite-nuclear-mass (INM) calculations. The $\alpha^{4}$ was estimated using the procedure also developed in those works of Pachucki and Komasa.

\section{METHOD USED IN THE CALCULATIONS}

In the first stage of the calculations we computed the nonrelativistic wave functions for the lowest two $S$ states of Li. In the calculations we used the Hamiltonian obtained by separating the center-of-mass motion from the nonrelativistic laboratory-frame Hamiltonian. This separation reduces the four-particle $\mathrm{Li}$ problem to a three-pseudoparticle problem with a Hamiltonian that we call internal $\left(\hat{H}_{\mathrm{INT}}\right) . H_{\mathrm{INT}}$ has the following form:

$$
\begin{aligned}
\hat{H}_{\mathrm{INT}}= & -\frac{1}{2}\left(\sum_{i=1}^{3} \frac{1}{\mu_{i}} \nabla_{\mathbf{r}_{i}}^{2}+\sum_{i=1}^{3} \sum_{j \neq i}^{3} \frac{1}{m_{0}} \nabla_{\mathbf{r}_{i}} \cdot \nabla_{\mathbf{r}_{j}}\right)+\sum_{i=1}^{3} \frac{q_{0} q_{i}}{r_{i}} \\
& +\sum_{i=1}^{3} \sum_{i<j}^{3} \frac{q_{i} q_{j}}{r_{i j}},
\end{aligned}
$$

where in atomic units $q_{0}=3$ (the charge of the nucleus), $q_{1}$ $=q_{2}=q_{3}=-1$ (the charges of the electrons), $m_{0}=12786.393$ (the mass of the nucleus) for the ${ }^{7} \mathrm{Li}$ nucleus and $m_{0}$ $=10961.898$ for the ${ }^{6} \mathrm{Li}$ nucleus, $\mu_{i}$ are the reduced electron masses, and $\mu_{i}=m_{0} m_{i} /\left(m_{0}+m_{i}\right)$, where $m_{1}=m_{2}=m_{3}=1 . \mathbf{r}_{i}$, $i=1,2,3$, are the position vectors of the electrons with respect to the nucleus, $r_{i}$ are their lengths, and $r_{i j}=\left|\mathbf{r}_{j}-\mathbf{r}_{i}\right|$ are the distances between the electrons. The calculations with the $\hat{H}_{\text {INT }}$ Hamiltonian have been carried out for finite and infinite masses of the Li nucleus. They yielded the nonrelativistic energies $E_{\mathrm{NR}}$ and the corresponding wave functions.

To describe the relativistic effects in the calculations we use the Dirac-Breit Hamiltonian in the Pauli approximation $\left(\hat{H}_{\text {rel }}\right)[15,32]$ transformed to the internal coordinate system. For states with the $S$ symmetry $\hat{H}_{\text {rel }}$ includes the massvelocity (MV), Darwin (D), orbit-orbit (OO), and spin-spin (SS) terms:

$$
\hat{H}_{\mathrm{rel}}=\hat{H}_{\mathrm{MV}}+\hat{H}_{\mathrm{D}}+\hat{H}_{\mathrm{OO}}+\hat{H}_{\mathrm{SS}} .
$$

The explicit form of these operators in the internal coordinates is

$$
\begin{gathered}
\hat{H}_{\mathrm{MV}}=-\frac{1}{8}\left[\frac{1}{m_{0}^{3}}\left(\sum_{i=1}^{3} \boldsymbol{\nabla}_{\mathbf{r}_{i}}\right)^{4}+\sum_{i=1}^{3} \frac{1}{m_{i}^{3}} \nabla_{\mathbf{r}_{i}}^{4}\right] \\
\hat{H}_{\mathrm{D}}=-\frac{1}{2} \pi\left[\sum_{i=1}^{3} \frac{q_{0} q_{i}}{m_{i}^{2}} \delta^{3}\left(\mathbf{r}_{i}\right)+\sum_{i=1}^{3} \sum_{j \neq i}^{3} \frac{q_{i} q_{j}}{m_{i}^{2}} \delta^{3}\left(\mathbf{r}_{i j}\right)\right], \\
\hat{H}_{\mathrm{OO}}=-\frac{1}{2} \sum_{i=1}^{3} \sum_{j=1}^{3} \frac{q_{0} q_{i}}{m_{0} m_{i}}\left[\frac{1}{r_{i}} \boldsymbol{\nabla}_{\mathbf{r}_{i}} \cdot \boldsymbol{\nabla}_{\mathbf{r}_{j}}+\frac{1}{r_{i}^{3}} \mathbf{r}_{i} \cdot\left(\mathbf{r}_{i} \cdot \boldsymbol{\nabla}_{\mathbf{r}_{i}}\right) \boldsymbol{\nabla}_{\mathbf{r}_{j}}\right] \\
+\frac{1}{2} \sum_{i=1}^{2} \sum_{j>i}^{3} \frac{q_{i} q_{j}}{m_{i} m_{j}}\left[\frac{1}{r_{i j}} \boldsymbol{\nabla}_{\mathbf{r}_{i}} \cdot \nabla_{\mathbf{r}_{j}}+\frac{1}{r_{i j}^{3}} \mathbf{r}_{i j} \cdot\left(\mathbf{r}_{i j} \cdot \nabla_{\mathbf{r}_{i}}\right) \boldsymbol{\nabla}_{\mathbf{r}_{j}}\right], \\
\hat{H}_{\mathrm{SS}}=-\frac{2}{3} \pi \sum_{i=1}^{3} \sum_{j>i}^{3} \frac{q_{i} q_{j}}{m_{i} m_{j}} \delta^{3}\left(\mathbf{r}_{i j}\right)\left(\mathbf{S}_{i} \cdot \mathbf{S}_{j}\right) \\
-\frac{2}{3} \pi \sum_{i=1}^{3} \frac{q_{0} q_{i}}{m_{0} m_{i}} \delta^{3}\left(\mathbf{r}_{i}\right)\left(\mathbf{S}_{0} \cdot \mathbf{S}_{i}\right) .
\end{gathered}
$$

In the present calculations we have not included the magnetic anomaly factors for the electrons and the nucleus in the Darwin and spin-spin corrections. This was done to make the comparison with the results obtained with the Hylleraas functions by Puchalski and Pachucki [20,23] more direct, since they also did not include the electronic and nuclear magnetic anomaly in their calculations. For the same reason we did not include a very small additional term proportional to the inverse square of the nucleus mass in the Darwin Hamiltonian that arises due to the interaction between the nucleus and the electrons (we included this term in one of our previous works [4]).

The calculations of the $\alpha^{2}$ relativistic corrections in this work have been performed by computing the expectation value of the $H_{\text {rel }}$ operator with the nonrelativistic FNM wave function-i.e., the wave functions obtained in the calculations with the finite masses of the ${ }^{6} \mathrm{Li}$ and ${ }^{7} \mathrm{Li}$ nuclei $\left(\Psi_{\mathrm{FNM}}\right)$. We also performed the calculations of the relativistic corrections with the wave function obtained in the infinite-mass calculation $\left(\Psi_{\text {INM }}\right)$. Since the procedure used in this work for calculating the $\alpha^{3}$ and $\alpha^{4}$ QED corrections was only developed for the infinite-mass wave function, only this type of calculations have been performed.

A nonrelativistic wave function that describes a stationary state of the Li atom (either $\Psi_{\mathrm{FNM}}$ or $\Psi_{\mathrm{INM}}$ ) is an antisymmetrized product of a function of the internal spatial coordinates $\mathbf{r}$ and of the spin coordinates $\boldsymbol{\sigma}$ :

$$
\Psi(\mathbf{r}, \sigma)=\hat{A}\left[\Phi(\mathbf{r}) \Omega_{S, M_{S}}(\sigma)\right] .
$$

The antisymmetrization operator $\hat{A}$ acts only on the electronic coordinates. The spin function $\Omega_{S, M_{S}}(\sigma)$ is a product of an electronic spin function and a nuclear spin function, $\Omega_{S, M_{S}}=\Omega^{e} \Omega^{N}$. For the two states of Li considered here we used the following electronic spin function: $\Omega^{e}$ $=(1 / \sqrt{6})[2 \alpha(1) \beta(2) \alpha(3)-\beta(1) \alpha(2) \alpha(3)-\alpha(1) \alpha(2) \beta(3)]$. 
In calculating the QED corrections we used the following approach. As was described in the work of Pachucki and Komasa [29], the leading QED correction for the Li atom that accounts for the two-photon exchange, the vacuum polarization, and the electron self-energy effects has the following form:

$$
\begin{aligned}
E_{\mathrm{QED}}= & \sum_{i=1}^{3} \sum_{j>i}^{3}\left\{\left[\frac{164}{15}+\frac{14}{3} \ln \alpha\right]\left\langle\Psi_{\mathrm{INM}}\left|\delta^{3}\left(\mathbf{r}_{i j}\right)\right| \Psi_{\mathrm{INM}}\right\rangle\right. \\
& \left.-\frac{14}{3}\left\langle\Psi_{\mathrm{INM}}\left|\frac{1}{4 \pi} P\left(\frac{1}{r_{i j}^{3}}\right)\right| \Psi_{\mathrm{INM}}\right\rangle\right\}+\sum_{i=1}^{3}\left[\frac{19}{30}-2 \ln \alpha\right. \\
& \left.-\ln k_{0}\right] \frac{4 q_{0}}{3}\left\langle\Psi_{\mathrm{INM}}\left|\delta^{3}\left(\mathbf{r}_{i}\right)\right| \Psi_{\mathrm{INM}}\right\rangle .
\end{aligned}
$$

The above equation excludes the recoil contributions which are usually much smaller than the main contributions. The last term in braces in expression (9) is the so called ArakiSucher distribution $[16,17,25,33]$, which is defined as the following limit:

$$
\begin{aligned}
\left\langle\Psi\left|P\left(\frac{1}{r^{3}}\right)\right| \Psi^{\prime}\right\rangle= & \lim _{a \rightarrow 0} \int \Psi^{*}(\mathbf{r}) \Psi^{\prime}(\mathbf{r})\left[\frac{1}{r^{3}} \Theta(r-a)\right. \\
& \left.+4 \pi \delta^{3}(\mathbf{r})(\gamma+\ln a)\right] d \mathbf{r},
\end{aligned}
$$

where $\Theta$ is the step function and $\gamma$ is the Euler constant. In calculating the distribution we employed the so-called expectation value identity approach [34], which allowed us to overcome the slow convergence of the highly singular $P\left(1 / r_{i j}^{3}\right)$ term. There is another term in expression (9) that is more difficult to calculate for an atom with more than one electron. It involves the so-called Bethe logarithm $\ln k_{0}$, defined as

$$
\begin{aligned}
\ln k_{0}= & -\frac{1}{D}\left\langle\Psi_{\mathrm{INM}}\right| \boldsymbol{\nabla}\left(\hat{H}_{\mathrm{INT}}-E_{\mathrm{NR}}\right) \ln \left[2 \left(\hat{H}_{\mathrm{INT}}\right.\right. \\
& \left.\left.-E_{\mathrm{NR}}\right)\right] \boldsymbol{\nabla}\left|\Psi_{\mathrm{INM}}\right\rangle,
\end{aligned}
$$

where for $\mathrm{Li}$

$$
\boldsymbol{\nabla}=\sum_{i=1}^{3} \boldsymbol{\nabla}_{i}
$$

and

$$
D=2 \pi q_{0}\left\langle\Psi_{\mathrm{INM}}\left|\sum_{i=1}^{3} \delta^{3}\left(\mathbf{r}_{i}\right)\right| \Psi_{\mathrm{INM}}\right\rangle .
$$

High-precision calculations of $\ln k_{0}$ have been done for some one- and two-electron atoms (see, e.g., $[35,36]$ ). Recently Yan and Drake [26] and Pachucki and Komasa [29] also reported high-quality QED results including $\ln k_{0}$ for the ground and first excited states of the lithium atom. Later Pachucki and Komasa also calculated the Bethe logarithm for the ground state of $\mathrm{Be}^{+}$and $\mathrm{Li}^{-}$and the ground and first excited states of $\mathrm{Be}[28,30]$. The evaluation of $\ln k_{0}$ in those works was based on the integral representation of $\ln k_{0}$ proposed by Schwartz [37] and reformulated in a more concise form by Pachucki and Komasa [30]. The calculation of $\ln k_{0}$ in the present work was done with the procedure developed by Pachucki and Komasa [30].

In the present work the calculations of the $\alpha^{4}$ QED correction have been done with an approximate procedure that includes only the dominant component of the correction. This component typically accounts for about $80 \%$ of the total value of the correction, and it is obtained from the following equation [30]:

$$
E_{\mathrm{HQED}} \simeq 4 \pi q_{0}^{2}\left(\frac{139}{128}+\frac{5}{192}-\frac{\ln 2}{2}\right)\left\langle\Psi_{\mathrm{INM}}\left|\sum_{i=1}^{3} \delta^{3}\left(\mathbf{r}_{i}\right)\right| \Psi_{\mathrm{INM}}\right\rangle .
$$

The remaining $\alpha^{4}$ contributions involve some singular terms that are more difficult to calculate $[38,39]$. These contributions were neglected in the present calculations.

The nonrelativistic wave functions for the two $S$ states of Li considered in this work have been expanded using the following ECG basis functions:

$$
\phi_{k}=\exp \left[-\mathbf{r}^{\prime}\left(A_{k} \otimes I_{3}\right) \mathbf{r}\right]=\exp \left[-\mathbf{r}^{\prime}\left(L_{k} L_{k}^{\prime} \otimes I_{3}\right) \mathbf{r}\right],
$$

where $\otimes$ is the Kronecker product symbol, $\mathbf{r}$ is a vector of the internal Cartesian coordinates, $\mathbf{r}_{1}, \mathbf{r}_{2}$, and $\mathbf{r}_{3}$, of the three pseudoelectrons ( $\mathbf{r}$ is a $9 \times 1$ vector), $L_{k}$ is a $3 \times 3$ lower triangular matrix of nonlinear variational parameters, and $I_{3}$ is the $3 \times 3$ identity matrix. The permutational symmetry of the wave function was implemented by applying symmetry projector $\hat{A}$ to each basis function.

The reason for representing the nonlinear parameters of the Gaussians (15) in the Cholesky factored form $L_{k} L_{k}^{\prime}$ is to avoid dealing with constrains that would otherwise need to be imposed if the parameters were represented by a single matrix $A_{k}$. Those constraints result from requiring that each basis function be square integrable, and this can only happen if $A_{k}$ is a positive-definite matrix. If $A_{k}=L_{k} L_{k}^{\prime}$ and $L_{k}$ is a lower triangular matrix, the square-integrability condition is automatically satisfied and no restrictions need to imposed on the elements of the $L_{k}$ matrix (they can vary in the range of $[-\infty,+\infty]$ ). This allows us to perform unrestricted optimization of $L_{k}$ 's, which is much simpler and more efficient than the optimization with constrains.

In the nonrelativistic calculations in this work we used the variational method. For a given basis set with size $K$ and the corresponding set of matrices of the nonlinear parameters $\left\{L_{k}, k=1, \ldots, K\right\}$, the nonrelativistic energy of the $i$ th state, $E^{i}$, and its wave function were obtained by solving the secular equation

$$
H c^{i}=E^{i} S c^{i},
$$

where $H$ and $S$ are the $K \times K$ Hamiltonian and overlap matrices, respectively (their elements depend on $L_{k}$ 's) and $c^{i}$ is the vector of the linear expansion coefficients $c_{k}^{i}$. In order to obtain highly accurate energies and wave functions, it is necessary to perform an extensive minimization of the energy with respect to the elements of the $L_{k}$ 's matrices. In this minimization we employed the analytic gradient of the energy with respect to those elements. To calculate the gradient 
one needs to determine the analytic derivatives of the $H\left(\left\{L_{k}\right\}\right)$ and $S\left(\left\{L_{k}\right\}\right)$ matrix elements. The formulas for these derivatives are implemented in our computer code. The use of the analytic gradient significantly accelerates the optimization process and allows us to achieve high accuracy at a relatively low computational cost.

In the present calculations, the variational optimization of the $L_{k}$ parameters was performed independently for each state. This was only done for the ${ }^{7} \mathrm{Li}$ isotop. In the calculations of the ${ }^{6} \mathrm{Li}$ isotop and in the ${ }^{\infty} \mathrm{Li}$ calculations, only the linear coefficients in the wave function were changed and the basis set used was the same as the one generated for the ${ }^{7} \mathrm{Li}$ isotop. Our previous atomic calculations have showed that, if the mass of the isotope changes, it is sufficient to only adjust the linear expansion coefficients of the wave function to account for the mass difference.

The numerical values of the fine-structure constant and the hartree- $\mathrm{MHz}$ conversion factor used in this work were taken from [40]:

$$
\alpha=1 / 137.035999679 \text {, }
$$

1 hartree $=6579683920.722 \mathrm{MHz}$

\section{RESULTS}

In the first step of the calculations we determined the nonrelativistic FNM variational wave functions and the total energy for the two lowest $S$ states of the Li atom considered in this work. In the calculations the basis set for each state has been grown to the size of 10000 functions. Nearly that many Hylleraas functions were used by Pachucki et al. and Yan et al. in their calculations on the two states of $\mathrm{Li}$ $[20,23,24,27]$. The growing of the basis set involved gradually adding subsets of 20 functions to the basis set and optimizing each function of the subset, one function at a time. After the addition of each 20 functions the entire basis set was reoptimized in a cyclic optimization where again the parameters of one function at a time were reoptimized using the gradient-based minimization procedure. A stochastic procedure was used to select the initial values of the nonlinear parameters of the added functions. The distribution of these values was chosen based on the parameters of the functions already included in the basis set. When the level of 10000 functions was reached for each state, several additional cyclic optimizations were performed of all the functions to get the final basis set. The calculations of the nonrelativistic energies and the corresponding wave functions constituted by far the most time-consuming step of this work. Even with the

TABLE I. Total nonrelativistic energies $E_{\mathrm{NR}}$, relativistic corrections, and total relativistic energies (the sum of $E_{\mathrm{NR}}$ and the relativistic corrections) for the $2{ }^{2} S_{1 / 2}$ and $3{ }^{2} S_{1 / 2}$ states of ${ }^{\infty} \mathrm{Li},{ }^{7} \mathrm{Li}$, and ${ }^{6} \mathrm{Li}$. The $\left\langle\hat{H}_{\mathrm{MV}}+\hat{H}_{\mathrm{D}}+\hat{H}_{\mathrm{SS}}\right\rangle$ and $\left\langle\hat{H}_{\mathrm{OO}}\right\rangle$ corrections are divided by $\alpha^{2}$. All quantities are in a.u.

\begin{tabular}{|c|c|c|c|c|c|c|c|}
\hline System & State & Basis & $E_{\mathrm{NR}}$ & $\left\langle\hat{H}_{\mathrm{MV}}+\hat{H}_{\mathrm{D}}+\hat{H}_{\mathrm{SS}}\right\rangle$ & $\left\langle\hat{H}_{\mathrm{OO}}\right\rangle$ & $\alpha^{2} E_{\text {rel }}$ & $E_{\mathrm{NR}}+\alpha^{2} E_{\mathrm{rel}}$ \\
\hline \multirow[t]{8}{*}{${ }^{\infty} \mathrm{Li}$} & \multirow[t]{4}{*}{$2{ }^{2} S_{1 / 2}$} & 7000 & -7.47806032324 & -11.6134382 & -0.4355980 & $-6.4162749 \times 10^{-4}$ & -7.47870195073 \\
\hline & & 8000 & -7.47806032354 & -11.6135228 & -0.4355979 & $-6.4163200 \times 10^{-4}$ & -7.47870195553 \\
\hline & & 9000 & -7.47806032371 & -11.6136752 & -0.4355979 & $-6.4164011 \times 10^{-4}$ & -7.47870196382 \\
\hline & & 10000 & -7.47806032381 & -11.6137653 & -0.4355979 & $-6.4164490 \times 10^{-4}$ & -7.47870196872 \\
\hline & \multirow[t]{4}{*}{$3{ }^{2} S_{1 / 2}$} & 7000 & -7.35409842020 & -11.4407368 & -0.4299087 & $-6.3212795 \times 10^{-4}$ & -7.35473054815 \\
\hline & & 8000 & -7.35409842073 & -11.4407120 & -0.4299087 & $-6.3212662 \times 10^{-4}$ & -7.35473054735 \\
\hline & & 9000 & -7.35409842095 & -11.4408337 & -0.4299087 & $-6.3213310 \times 10^{-4}$ & -7.35473055406 \\
\hline & & 10000 & -7.35409842113 & -11.4408183 & -0.4299087 & $-6.3213229 \times 10^{-4}$ & -7.35473055342 \\
\hline \multirow[t]{8}{*}{${ }^{7} \mathrm{Li}$} & \multirow[t]{4}{*}{$2{ }^{2} S_{1 / 2}$} & 7000 & -7.47745193011 & -11.6051358 & -0.4454912 & $-6.4171221 \times 10^{-4}$ & -7.47809364232 \\
\hline & & 8000 & -7.47745193041 & -11.6052204 & -0.4454912 & $-6.4171671 \times 10^{-4}$ & -7.47809364711 \\
\hline & & 9000 & -7.47745193058 & -11.6053727 & -0.4454911 & $-6.4172481 \times 10^{-4}$ & -7.47809365540 \\
\hline & & 10000 & -7.47745193068 & -11.6054627 & -0.4454911 & $-6.4172961 \times 10^{-4}$ & -7.47809366029 \\
\hline & \multirow[t]{4}{*}{$3{ }^{2} S_{1 / 2}$} & 7000 & -7.35350048700 & -11.4325631 & -0.4396995 & $-6.3221406 \times 10^{-4}$ & -7.35413270106 \\
\hline & & 8000 & -7.35350048752 & -11.4325384 & -0.4396995 & $-6.3221274 \times 10^{-4}$ & -7.35413270027 \\
\hline & & 9000 & -7.35350048775 & -11.4326600 & -0.4396995 & $-6.3221922 \times 10^{-4}$ & -7.35413270697 \\
\hline & & 10000 & -7.35350048793 & -11.4326447 & -0.4396995 & $-6.3221840 \times 10^{-4}$ & -7.35413270633 \\
\hline \multirow[t]{8}{*}{${ }^{6} \mathrm{Li}$} & \multirow{4}{*}{$2{ }^{2} S_{1 / 2}$} & 7000 & -7.47735068068 & -11.6034405 & -0.4471373 & $-6.4170959 \times 10^{-4}$ & -7.47799239026 \\
\hline & & 8000 & -7.47735068097 & -11.6035250 & -0.4471373 & $-6.4171409 \times 10^{-4}$ & -7.47799239506 \\
\hline & & 9000 & -7.47735068115 & -11.6036773 & -0.4471372 & $-6.4172219 \times 10^{-4}$ & -7.47799240334 \\
\hline & & 10000 & -7.47735068125 & -11.6037673 & -0.4471372 & $-6.4172699 \times 10^{-4}$ & -7.47799240823 \\
\hline & \multirow[t]{4}{*}{$3{ }^{2} S_{1 / 2}$} & 7000 & -7.35340097820 & -11.4308790 & -0.4413286 & $-6.3221113 \times 10^{-4}$ & -7.35403318934 \\
\hline & & 8000 & -7.35340097873 & -11.4308543 & -0.4413286 & $-6.3220982 \times 10^{-4}$ & -7.35403318854 \\
\hline & & 9000 & -7.35340097895 & -11.4309759 & -0.4413286 & $-6.3221629 \times 10^{-4}$ & -7.35403319525 \\
\hline & & 10000 & -7.35340097913 & -11.4309606 & -0.4413286 & $-6.3221547 \times 10^{-4}$ & -7.35403319461 \\
\hline
\end{tabular}


TABLE II. Literature values of nonrelativistic energies and relativistic corrections for $2{ }^{2} S_{1 / 2}$ and $3{ }^{2} S_{1 / 2}$ states of ${ }^{\infty}$ Li computed with Hylleraas-type basis functions.

\begin{tabular}{llcccccc}
\hline \hline State & \multicolumn{1}{c}{ Basis } & $E_{\mathrm{NR}}$ & $\left\langle\hat{H}_{\mathrm{MV}}+\hat{H}_{\mathrm{D}}+\hat{H}_{\mathrm{SS}}\right\rangle$ & $\left\langle\hat{H}_{\mathrm{OO}}\right\rangle$ & $\alpha^{2} E_{\mathrm{rel}}$ & $E_{\mathrm{NR}}+\alpha^{2} E_{\mathrm{rel}}$ & $\mathrm{Ref}$. \\
\hline $2{ }^{2} S_{1 / 2}$ & 9576 & -7.4780603238897 & -11.6143159 & -0.4355979 & $-6.4167422 \times 10^{-4}$ & -7.47870199811 & {$[23]$} \\
$2{ }^{2} S_{1 / 2}$ & $\infty$ (extrap.) & -7.4780603239041 & -11.6143001 & -0.4355978 & $-6.4167338 \times 10^{-4}$ & -7.47870199728 & {$[23]$} \\
$2{ }^{2} S_{1 / 2}$ & $\infty$ (extrap.) & -7.4780603236503 & -11.6143119 & -0.4355980 & $-6.4167402 \times 10^{-4}$ & -7.47870199767 & {$[25]$} \\
$2{ }^{2} S_{1 / 2}$ & 9577 & -7.4780603238924 & & & & \\
$3{ }^{2} S_{1 / 2}$ & 9576 & -7.3540984213799 & -11.4412626 & -0.4299088 & $-6.3215596 \times 10^{-4}$ & -7.35473057734 & {$[24]$} \\
$3{ }^{2} S_{1 / 2}$ & $\infty$ (extrap.) & -7.354098421407 & -11.4412596 & -0.4299087 & $-6.3215579 \times 10^{-4}$ & -7.35473057719 & {$[24]$} \\
\hline \hline
\end{tabular}

use of a parallel supercomputer system (in most calculations we either used 8 or 16 processors) they lasted several months of continuous computing. In Table I we show how the total nonrelativistic FNM energies for each of the two states vary when the basis set size changes from 7000 to 10000 in increments of 1000 . We also show the energies obtained with setting the mass of the Li nucleus to infinity (the INM calculations). As one can see, for both states with 10000 functions the nonrelativistic energy is converged to the relative accuracy of $10^{-10}$, if not higher.

In the next step the nonrelativistic FNM wave functions generated with the procedure described above were used to calculate the $\alpha^{2}$ relativistic corrections-i.e., the MV, Darwin, spin-spin interaction, and orbit-orbit corrections. The expectation values of the sum of the MV, Darwin, and spinspin interaction Hamiltonians $\left(\left\langle\hat{H}_{\mathrm{MV}}+\hat{H}_{\mathrm{D}}+\hat{H}_{\mathrm{SS}}\right\rangle\right)$, as well as the orbit-orbit interaction Hamiltonian $\left(\left\langle\hat{H}_{\mathrm{OO}}\right\rangle\right)$, are shown in Table I. The values shown were obtained for ${ }^{\infty} \mathrm{Li},{ }^{6} \mathrm{Li}$, and ${ }^{7} \mathrm{Li}$ with the 7000-term, 8000-term, 9000-term, and 10000 term wave functions. We also show the total relativistic correction multiplied by $\alpha^{2}$ and the total energy that includes the nonrelativistic part and the $\alpha^{2}$ relativistic correction. These results allow one to assess the level of convergence of the two components of the energy with the basis set size.

As one can see upon examining the results in Table I, while the ninth to tenth digit after the decimal point in the nonrelativistic energy is essentially converged, the convergence level of the relativistic correction is somewhat lower. This is clearly related to the presence of highly singular op- erators, such as the fourth powers of the linear momentum operator (in the MV correction) and three-dimensional Dirac $\Delta$ functions dependent on the interparticle distances (in the D and SS corrections), in the relativistic corrections. This is also related to the less efficient representation of the cusp conditions by the Gaussian basis functions in comparison with, for example, the Hylleraas functions. However, the absolute convergence of about $10^{-8}$ a.u. of the relativistic correction for the two states considered in this work should be sufficient to determine the $3{ }^{2} S_{1 / 2} \rightarrow 2{ }^{2} S_{1 / 2}$ transition energy with adequate accuracy.

The results for ${ }^{\infty} \mathrm{Li}$ shown in Table I allow a direct comparison with the results obtained by Puchalski and Pachucki $[23,24]$ and by Yan et al. $[25,27]$. This comparison is shown in Table II. Our best nonrelativistic energy for the ground state of -7.47806032381 a.u. obtained with 10000 basis functions is only $8 \times 10^{-11}$ a.u. higher than the best energy of -7.4780603238924 a.u. obtained by Yan et al. and the best energy of -7.4780603238897 a.u. obtained by Puchalski and Pachucki with 9577 and 9576 Hylleraas functions, respectively. For the first excited state our lowest energy obtained with 10000 basis functions of -7.35409842112 a.u. is also slightly higher than the best energy of Puchalski and Pachucki of -7.3540984213799 a.u. We estimate that it would take 12 000-14 000 Gaussians to converge our nonrelativistic calculations to the results obtained in [23,24,27]. Even though we could have continued to increase the basis set size for each state beyond 10000 functions - there were no software or hardware limits preventing this—we decided to stop at 10000 because that was about the same number of

TABLE III. $3{ }^{2} S_{1 / 2} \rightarrow 2{ }^{2} S_{1 / 2}$ transition energies for ${ }^{\infty} \mathrm{Li},{ }^{7} \mathrm{Li}$, and ${ }^{6} \mathrm{Li}$ calculated with Gaussians using only nonrelativistic total energies $E_{\mathrm{NR}}$ (denoted as $\mathrm{nr}$ ) and relativistic total energies $E_{\mathrm{NR}}+\alpha^{2}: E_{\mathrm{rel}}$ (denoted as rel). For comparison we show the results obtained with the Hylleraas-type basis functions by extrapolating to the limit of infinite basis set (the value is mainly derived from the data reported in [24], while relativistic recoil corrections are taken from [26]). All energies are in $\mathrm{MHz}$.

\begin{tabular}{|c|c|c|c|c|c|c|}
\hline \multirow[b]{2}{*}{ Basis } & \multicolumn{2}{|c|}{${ }^{\infty} \mathrm{Li}$} & \multicolumn{2}{|c|}{${ }^{7} \mathrm{Li}$} & \multicolumn{2}{|c|}{${ }^{6} \mathrm{Li}$} \\
\hline & $\mathrm{nr}$ & rel & $\mathrm{nr}$ & rel & $\mathrm{nr}$ & rel \\
\hline 7000 & 815630140.23 & 815692644.24 & 815561317.22 & 815623812.02 & 815549864.35 & 815612361.18 \\
\hline 8000 & 815630138.71 & 815692681.06 & 815561315.71 & 815623848.77 & 815549862.84 & 815612397.92 \\
\hline 9000 & 815630138.36 & 815692691.46 & 815561315.36 & 815623859.17 & 815549862.48 & 815612408.33 \\
\hline 10000 & 815630137.85 & 815692727.86 & 815561314.84 & 815623895.56 & 815549861.97 & 815612444.71 \\
\hline Literature & 815630136.73 & 815692763.23 & 815561313.73 & 815623939.10 & 815549860.86 & 815612486.04 \\
\hline
\end{tabular}


TABLE IV. Shifts of the nonrelativistic and relativistic contributions to the transition energy due to the finite nuclear mass effect. All values are in $\mathrm{MHz}$.

\begin{tabular}{lcccc}
\hline \hline Basis & $\Delta E_{\mathrm{NR}}\left({ }^{7} \mathrm{Li}-{ }^{\infty} \mathrm{Li}\right)$ & $\Delta E_{\mathrm{NR}}\left({ }^{6} \mathrm{Li}-{ }^{\infty} \mathrm{Li}\right)$ & $\alpha^{2} \Delta E_{\text {rel }}\left({ }^{7} \mathrm{Li}-{ }^{\infty} \mathrm{Li}\right)$ & $\alpha^{2} \Delta E_{\text {rel }}\left({ }^{6} \mathrm{Li}-{ }^{\infty} \mathrm{Li}\right)$ \\
\hline 7000 & -68823.00 & -80275.88 & 9.22 & 7.19 \\
8000 & -68823.00 & -80275.88 & 9.29 & 7.26 \\
9000 & -68823.00 & -80275.88 & 9.28 & 7.25 \\
10000 & -68823.00 & -80275.88 & 9.30 & 7.28 \\
\hline \hline
\end{tabular}

functions used by Pachucki et al. and Yan et al. in their calculations $[20,23,24,27]$. Having the results obtained with about the same number of Gaussians as they had Hylleraas functions enabled a direct comparison of the efficiency of the two types of functions in describing the two lowest $S$ states of the $\mathrm{Li}$ atom.

The comparison of the $\alpha^{2}$ relativistic corrections obtained in the present calculations and those obtained by Yan and Drake [25] and Puchalski and Pachucki [23,24] shows that even with 10000 Gaussians our results are not as tightly converged as theirs. This particularly applies to the $\left\langle\hat{H}_{\mathrm{MV}}\right.$ $\left.+\hat{H}_{\mathrm{D}}+\hat{H}_{\mathrm{SS}}\right\rangle$ expectation value. For the ground state we get $-11.6137653 \alpha^{2}$ a.u. for this quantity with 10000 Gaussians, while the Yan-Drake and Puchalski-Pachucki values are $-11.6143119 \alpha^{2}$ a.u. and $-11.6143159 \alpha^{2}$ a.u., respectively. At 10000 Gaussians our value is still changing in the fourth digit after the decimal point and moving towards the value of $-11.6143001 \alpha^{2}$ a.u. that Puchalski and Pachucki obtained by extrapolating their results to the infinite-basis-size limit. However, the convergence is slow. Much better converged is the orbit-orbit interaction correction $\left\langle\hat{H}_{\mathrm{OO}}\right\rangle$. With 10000 functions for the ground state we get $-0.435597912 \alpha^{2}$ a.u., while the Puchalski-Pachucki value is $-0.435597911 \alpha^{2}$ a.u. and the Yan-Drake value is $-0.435598001 \alpha^{2}$ a.u.

The comparison that most clearly reveals the quality of the calculations with Gaussians versus the calculations with the Hylleraas functions concerns the $3{ }^{2} S_{1 / 2} \rightarrow 2{ }^{2} S_{1 / 2}$ transition energy. This comparison is shown in Table III. The energy unit used is MHz. At the nonrelativistic level our transition energies obtained with 10000 Gaussians for ${ }^{\infty} \mathrm{Li},{ }^{7} \mathrm{Li}$, and ${ }^{6} \mathrm{Li}$ are only about $1 \mathrm{MHz}$ higher than the result of $\mathrm{Pu}-$ chalski and Pachucki obtained with 9576 Hylleraas functions. We should mention that, while in our approach the finite-mass effect is directly included in the variational nonrelativistic calculations, in their approach it is determined as a sum of the first- and second-order recoil corrections (i.e., corrections due to the finite electron to nucleus mass ratio).
With the relativistic corrections included in the energy our transition energy calculated for ${ }^{\infty} \mathrm{Li}$ starts to differ from the results of Puchalski and Pachucki by about $33 \mathrm{MHz}$. The difference increases to $44-41 \mathrm{MHz}$ for ${ }^{7} \mathrm{Li}$ and ${ }^{6} \mathrm{Li}$. This effect is likely related to the $\left\langle\hat{H}_{\mathrm{MV}}+\hat{H}_{\mathrm{D}}+\hat{H}_{\mathrm{SS}}\right\rangle$ expectation value not being as well converged in our calculations with Gaussians as it was with the Hylleraas functions. The slow convergence of $\left\langle\hat{H}_{\mathrm{MV}}+\hat{H}_{\mathrm{D}}+\hat{H}_{\mathrm{SS}}\right\rangle$ at present time remains the most significant limiting factor in our calculations.

The present FNM and IFM calculations allow an analysis of the mass effect on the nonrelativistic and relativistic contributions to the $3{ }^{2} S_{1 / 2} \rightarrow 2{ }^{2} S_{1 / 2}$ transition energy. These effects can be calculated as the differences of the respective FNM and IFM transition energy contributions. By explicitly including in the calculations of the nonrelativistic energy and the relativistic correction the finite nuclear mass, we can directly determine the FNM effects. The results obtained for the basis set sizes ranging from 7000 to 10000 are shown in Table IV. As one can see, for both Li isotops the mass nonrelativistic and relativistic contributions to the transition energies appear to be sufficiently converged with the number of the basis functions. The much larger of the two contributions, the nonrelativistic contribution, is, as expected, higher for ${ }^{6} \mathrm{Li}$ than for ${ }^{7} \mathrm{Li}(-80275.88 \mathrm{MHz}$ vs -68 823.00 MHz). The opposite is the case for the much smaller relativistic contribution, which is slightly higher for ${ }^{7} \mathrm{Li}$ than for ${ }^{6} \mathrm{Li}$ (9.30 MHz vs $7.28 \mathrm{MHz}$ ). It is interesting that the FNM correction to the relativistic contribution to the transition energy for each $\mathrm{Li}$ isotope determined here is more than 10 times higher than the corresponding correction-i.e., recoil correction-determined by Puchalski [24] using the perturbation approach (we should mention that the result reported by Puchalski includes recoil corrections to the relativistic and QED contributions). At present we do not have an explanation for this discrepancy.

The next step of the calculations involved determination of the leading $\alpha^{3}$ and $\alpha^{4}$ QED corrections. Apart from the values of the corrections we also show in Table $\mathrm{V}$ the values

TABLE V. $\alpha^{3}$ and $\alpha^{4}$ QED corrections ( $\alpha^{3} E_{\mathrm{QED}}$ and $\left.\alpha^{4} E_{\mathrm{HQED}}\right)$ for the two lowest doublet $S$ states of Li obtained in the infinite-mass calculations. The Araki-Sucher term, Eq. (10), and the Bethe logarithm, Eq. (11), are also shown. All values are in a.u.

\begin{tabular}{lccccc}
\hline \hline State & $\left\langle P\left(1 / r_{i j}^{3}\right)\right\rangle /(4 \pi)$ & $\ln k_{0}$ & $\alpha^{3} E_{\mathrm{QED}}$ & $\alpha^{4} E_{\mathrm{HQED}}$ & Ref. \\
\hline $2{ }^{2} S_{1 / 2}$ & 0.021767 & 5.17816 & $1.1136114 \times 10^{-4}$ & $3.39800 \times 10^{-6}$ & This work \\
$3{ }^{2} S_{1 / 2}$ & 0.015770 & 5.17935 & $1.1051117 \times 10^{-4}$ & $3.37195 \times 10^{-6}$ & This work \\
$2{ }^{2} S_{1 / 2}$ & & $5.17828(1)$ & & & {$[27]$} \\
$3{ }^{2} S_{1 / 2}$ & & $5.17958(6)$ & & & {$[27]$} \\
\hline \hline
\end{tabular}


TABLE VI. $3{ }^{2} S_{1 / 2} \rightarrow 2{ }^{2} S_{1 / 2}$ transition energy for ${ }^{7} \mathrm{Li}$ and ${ }^{6} \mathrm{Li}$ computed in this work with 10000 Gaussians after including relativistic (rel), $\alpha^{3}$ QED (QED), and $\alpha^{4}$ QED (HQED) corrections. For comparison we show the same quantity computed with Hylleraas-type basis functions by Puchalski and Pachucki [24], the transition energy for ${ }^{7} \mathrm{Li}$ computed with Hylleraas-type basis functions by Yan et al. [27], which also includes finite-nuclear-size correction (NUC), and the experimental transition energies by Bushaw et al. [41]. All values are in $\mathrm{MHz}$.

\begin{tabular}{|c|c|c|}
\hline & ${ }^{7} \mathrm{Li}$ & ${ }^{6} \mathrm{Li}$ \\
\hline $\mathrm{NR}+\mathrm{rel}+\mathrm{QED}+\mathrm{HQED}$, this work & 815618131.62 & 815606680.77 \\
\hline $\mathrm{NR}+\mathrm{rel}+\mathrm{QED}+\mathrm{HQED}[24]$ & 815618132.53 & 815606679.66 \\
\hline $\mathrm{NR}+\mathrm{rel}+\mathrm{QED}+\mathrm{HQED}+\mathrm{NUC}$ [27] & 815618149.30 & \\
\hline Experiment $[41]$ & $815618185.3(3.0)$ & $815606731.4(3.0)$ \\
\hline
\end{tabular}

of $P\left(1 / r_{i j}^{3}\right)$ and $\ln k_{0}$, which are the most difficult to compute. For the discussion on the accuracy of the procedure to calculate the QED corrections, we refer the reader to the work where the approach employed here was used in the calculations of the lowest excitation energy in the Be atom [31]. As one can see from the results presented in Table $\mathrm{V}$, the $\alpha^{3}$ and $\alpha^{4}$ corrections are by a factor of 6 and by a factor 200 , respectively, smaller than the $\alpha^{2}$ relativistic correction.

In the last step of this work we compare our transition energies obtained for ${ }^{7} \mathrm{Li}$ and ${ }^{6} \mathrm{Li}$ using the total energies of the two lowest $S$ states of these systems that include both the relativistic and QED corrections with the results obtained with the Hylleraas functions and with the experimental results. The results of this comparison are presented in Table VI. The difference between our transition energies and those by Puchalski and Pachucki [24] is only around $1 \mathrm{MHz}$. It should be said that such an excellent agreement is probably somewhat accidental. As was discussed previously, the discrepancy due to the relativistic corrections alone is 40 times larger than the value of $1 \mathrm{MHz}$. The reason why our value is so close to that one of Puchalski and Pachucki is that the discrepancy due to the relativistic corrections is almost entirely compensated by the discrepancy due to the QED corrections.

Even without taking into consideration the cancellation of the discrepancies in the final transition energy value, we can say that the level of accuracy that can be reached in calculations of small atoms, such as Li atoms, using Gaussian basis functions is quite satisfactory. These discrepancies are comparable in magnitude to the finite-nuclear-size effects and to the remaining difference between today's most accurate theoretical and experimental values of the $3{ }^{2} S_{1 / 2} \rightarrow 2{ }^{2} S_{1 / 2}$ transition energy. Further improvement in the calculations achieved by increasing the number Gaussian basis functions and more accurate determination of the expectation values of the singular operators can further reduce the discrepancies.

\section{SUMMARY}

In this work we have presented a series of calculations aimed to test the performance of explicitly correlated Gauss- ian functions in very accurate atomic calculations. The test concerned the lowest $S$-S transition energy of the ${ }^{7} \mathrm{Li}$ and ${ }^{6} \mathrm{Li}$ isotops of the $\mathrm{Li}$ atom. The energies of the two $S$ states were obtained by adding the relativistic and QED corrections calculated using the first-order perturbation theory to the nonrelativistic energies obtained in variational calculations. The nonrelativistic calculations have been performed using the nonrelativistic Schrödinger equation, which explicitly includes the nuclear motion. This is different from the traditional approach where the nonrelativistic infinite-nuclearmass energy is calculated first and then corrections due to the finite nucleus mass are added. The sum of the nonrelativistic FNM energy and the relativistic and QED corrections constituted the final total energy for each state that were used to calculate the $3{ }^{2} S_{1 / 2} \rightarrow 2{ }^{2} S_{1 / 2}$ transition energy.

The $3{ }^{2} S_{1 / 2} \rightarrow 2{ }^{2} S_{1 / 2}$ transition energies obtained in the calculations employing Gaussians are in a good agreement with those obtained with the Hylleraas functions. This is quite encouraging, considering that our goal is to use Gaussians and the approach presented here to perform very accurate calculations on atomic systems with more than three electrons. At present, Gaussians are the only choice of the basis functions for such calculations because the Hylleraastype and Slater-type functions have not yet been implemented for atomic systems with more than three electrons.

\section{ACKNOWLEDGMENTS}

This work has been supported by the National Science Foundation. We also acknowledge partial support of this work by a grant from the Polish Ministry of Science and Higher Education, No. N202 041 32/1045. We are grateful to the University of Arizona Center of Computing and Information Technology for the use of their supercomputer resources. J.K. acknowledges a generous computer time allotment from the Poznań Supercomputing and Networking Center. 
[1] M. Stanke, D. Kędziera, S. Bubin, and L. Adamowicz, J. Chem. Phys. 126, 194312 (2007).

[2] M. Stanke, D. Kȩdziera, S. Bubin, and L. Adamowicz, Phys. Rev. A 75, 052510 (2007).

[3] M. Stanke, D. Kȩdziera, S. Bubin, and L. Adamowicz, Phys. Rev. Lett. 99, 043001 (2007).

[4] M. Stanke, D. Kȩdziera, S. Bubin, and L. Adamowicz, J. Chem. Phys. 127, 134107 (2007).

[5] M. Stanke, J. Komasa, D. Kȩdziera, S. Bubin, and L. Adamowicz, Phys. Rev. A 77, 062509 (2008).

[6] M. Cafiero, S. Bubin, and L. Adamowicz, Phys. Chem. Chem. Phys. 5, 1491 (2003).

[7] S. Bubin, M. Cafiero, and L. Adamowicz, Adv. Chem. Phys. 131, 377 (2005).

[8] D. B. Kinghorn and L. Adamowicz, J. Chem. Phys. 110, 7166 (1999).

[9] D. B. Kinghorn and L. Adamowicz, Phys. Rev. Lett. 83, 2541 (1999).

[10] S. Bubin and L. Adamowicz, J. Chem. Phys. 118, 3079 (2003).

[11] S. Bubin, L. Adamowicz, and M. Molski, J. Chem. Phys. 123, 134310 (2005).

[12] W. E. Caswell and G. P. Lepage, Phys. Lett. 167B, 437 (1986).

[13] J. Soto, Eur. Phys. J. A 31, 705 (2007).

[14] J. Sapirstein, Int. J. Quantum Chem. 106, 3178 (2006).

[15] H. A. Bethe and E. E. Salpeter, Quantum Mechanics Of OneAnd Two-Electron Atoms (Plenum Publishing Corporation, New York, 1957).

[16] H. Araki, Prog. Theor. Phys. 17, 619 (1957).

[17] J. Sucher, Phys. Rev. 109, 1010 (1958).

[18] K. Pachucki, J. Phys. B 31, 3547 (1998).

[19] K. Pachucki, Phys. Rev. A 71, 012503 (2005).

[20] M. Puchalski, A. M. Moro, and K. Pachucki, Phys. Rev. Lett. 97, 133001 (2006).
[21] K. Pachucki, Phys. Rev. A 66, 062501 (2002).

[22] F. W. King, Phys. Rev. A 76, 042512 (2007).

[23] M. Puchalski and K. Pachucki, Phys. Rev. A 73, 022503 (2006).

[24] M. Puchalski, Ph.D. dissertation, Warsaw University, 2006.

[25] Z.-C. Yan and G. W. F. Drake, Phys. Rev. Lett. 81, 774 (1998).

[26] Z.-C. Yan and G. W. F. Drake, Phys. Rev. Lett. 91, 113004 (2003).

[27] Z.-C. Yan, W. Nörtershäuser, and G. W. F. Drake, Phys. Rev. Lett. 100, 243002 (2008)

[28] K. Pachucki and J. Komasa, J. Chem. Phys. 125, 204304 (2006).

[29] K. Pachucki and J. Komasa, Phys. Rev. A 68, 042507 (2003).

[30] K. Pachucki and J. Komasa, Phys. Rev. Lett. 92, 213001 (2004).

[31] K. Pachucki and J. Komasa, Phys. Rev. A 73, 052502 (2006).

[32] A. I. Akhiezer and V. B. Berestetskii, Quantum Electrodynamics (Interscience, New York, 1965).

[33] K. Pachucki, J. Phys. B 31, 5123 (1998).

[34] K. Pachucki, W. Cencek, and J. Komasa, J. Chem. Phys. 122, 184101 (2005)

[35] G. W. F. Drake, in Long-Range Casimir Forces: Theory and Recent Experiments on Atomic Systems, edited by F. S. Levine and D. A. Micha (Plenum Press, New York, 1993), p. 107.

[36] V. I. Korobov and S. V. Korobov, Phys. Rev. A 59, 3394 (1999).

[37] C. Schwartz, Phys. Rev. 123, 1700 (1961).

[38] V. Korobov and A. Yelkhovsky, Phys. Rev. Lett. 87, 193003 (2001).

[39] K. Pachucki, Phys. Rev. A 74, 022512 (2006).

[40] NIST reference on fundamental physical constants, http:// physics.nist.gov/cuu

[41] B. A. Bushaw, W. Nörtershäuser, G. Ewald, A. Dax, and G. W. F. Drake, Phys. Rev. Lett. 91, 043004 (2003). 